\title{
Orientational dynamics and energy landscape features of thermotropic liquid crystals: An analogy with supercooled liquids
}

\author{
BIMAN JANA and BIMAN BAGCHI* \\ Solid State and Structural Chemistry Unit, Indian Institute of Science, Bangalore 560012 \\ e-mail: bbagchi@sscu.iisc.ernet.in
}

MS received 15 June 2007; accepted 1 August 2007

\begin{abstract}
Recent optical kerr effect (OKE) studies have revealed that orientational relaxation of rodlike nematogens near the isotropic-nematic (I-N) phase boundary and also in the nematic phase exhibit temporal power law decay at intermediate times. Such behaviour has drawn an intriguing analogy with supercooled liquids. Here, we have investigated the single-particle and collective orientational dynamics of a family of model system of thermotropic liquid crystals using extensive computer simulations. Several remarkable features of glassy dynamics are on display including non-exponential relaxation, dynamical heterogeneity, and non-Arrhenius temperature dependence of the orientational relaxation time. Over a temperature range near the I-N phase boundary, the system behaves like a fragile glass-forming liquid. Using proper scaling, we construct the usual relaxation time versus inverse temperature plot and explicitly demonstrate that one can successfully define a density dependent fragility of liquid crystals. The fragility of liquid crystals shows a temperature and density dependence which is remarkably similar to the fragility of glass forming supercooled liquids. Energy landscape analysis of inherent structures shows that the breakdown of the Arrhenius temperature dependence of relaxation rate occurs at a temperature that marks the onset of the growth of the depth of the potential energy minima explored by the system.
\end{abstract}

Keywords. Liquid crystals; supercooled liquid; power law relaxation; fragility; heterogeneous dynamics; energy landscape.

\section{Introduction}

Thermotropic liquid crystals exhibit exotic phase behaviour upon temperature variation. In the isotropic phase, a liquid does not exhibit any long range translational or orientational order. The nematic phase is endowed with a long-ranged orientational order but lacks translational order. Further cooling leads to a more ordered smectic phase where two-dimensional translational order along with long-ranged orientational order sets in the system. The isotropic-nematic (I-N) phase transition, which is believed to be weakly first order in nature with certain characteristics of the continuous transition, has been a subject of immense attention in condensed matter physics and material sciences. ${ }^{1,2}$ In contrast, the dynamics of thermotropic liquid crystals have been much less studied, the focus being mostly on the long-time behaviour of orientational relaxation near the I-N transition. ${ }^{1}$ A series of OKE measurements have, however, recently studied

*For correspondence collective orientational relaxation in the isotropic phase near the I-N transition over a wide range of time scales. ${ }^{3,4}$ The dynamics have been found to be surprisingly rich, the most intriguing feature being the power law decay of the OKE signal at short-tointermediate times. ${ }^{3,4}$ The relaxation scenario appears to be strikingly similar to that of supercooled molecular liquids ${ }^{5}$, even though the latter do not undergo any thermodynamic phase transition.

To this end, we have undertaken molecular dynamics simulations of a family of model systems consisting of rod-like molecules across the I-N transition. Given the involvement of the phase transition to an orientationally ordered mesophase upon lowering the temperature, we choose to probe the single-particle and collective orientational dynamics in order to make comparison with relaxation behaviour observed for supercooled liquids. We have calculated the nonGaussian parameter in the orientational degrees of freedom in order to probe the heterogeneous dynamics present in the system near $\mathrm{I}-\mathrm{N}$ transition. We have defined a fragility index to quantitatively measure 
the glassy dynamics observed in the orientational degrees of freedom. We have also explored plausible correlation of the features of the underlying energy landscape with the observed non-Arrhenius dynamics in analogy with supercooled liquids.

\section{Models and simulation details}

The systems we have studied consist of ellipsoids of revolution. The Gay-Berne (GB) pair potential, ${ }^{6}$ that is well established to serve as a model potential for systems of thermotropic liquid crystals, has been employed. The GB pair potential, which uses a singlesite representation for each ellipsoid of revolution, is an elegant generalization of the extensively used isotropic Lennard-Jones potential to incorporate anisotropy in both the attractive and the repulsive parts of the interaction. ${ }^{6,7}$ In the GB pair potential, $i$ th ellipsoid of revolution is represented by the position $r_{i}$ of its center of mass and a unit vector $e_{i}$ along the long axis of the ellipsoid. The interaction potential between two ellipsoids of revolution $i$ and $j$ is given by

$$
U_{i j}^{G B}=4 \varepsilon\left(\hat{r}_{i j}, e_{i}, e_{j}\right)\left(\rho_{i j}^{-12}-\rho_{i j}^{-6}\right),
$$

where

$$
\rho_{i j}=\frac{r_{i j}-\sigma\left(\hat{r}_{i j}, e_{i}, e_{j}\right)+\sigma_{s s}}{\sigma_{s s}} .
$$

Here $\sigma_{s s}$ defines the thickness or equivalently, the separation between the two ellipsoids of revolution in a side-by-side configuration, $r_{i j}$ is the distance between the centers of mass of the ellipsoids of revolution $i$ and $j$, and $\hat{r}_{i j}=r_{i j} / r_{i j}$ is a unit vector along the intermolecular separation vector $r_{i j}$. The molecular shape parameter $\sigma$ and the energy parameter $\varepsilon$ both depend on the unit vectors $e_{i}$ and $e_{j}$ as well as on $\hat{r}_{i j}$ as given by the following set of equations:

$$
\begin{aligned}
& \sigma_{i j}\left(\hat{r}_{i j}, e_{i}, e_{j}\right)= \\
& \sigma_{0}\left[1-\frac{\chi}{2}\left\{\frac{\left(e_{i} \cdot \hat{r}_{i j}+e_{j} \cdot \hat{r}_{i j}\right)^{2}}{1+\chi\left(e_{i} \cdot e_{j}\right)}-\frac{\left(e_{i} \cdot \hat{r}_{i j}-e_{j} \cdot \hat{r}_{i j}\right)^{2}}{1-\chi\left(e_{i} \cdot e_{j}\right)}\right\}\right]^{-1 / 2}
\end{aligned}
$$

with $\chi=\left(\kappa^{2}+1\right) /\left(\kappa^{2}-1\right)$ and

$$
\varepsilon\left(\hat{r}_{i j}, e_{i}, e_{j}\right)=\varepsilon_{0}\left[\varepsilon_{1}\left(e_{i}, e_{j}\right)\right]^{\nu}\left[\varepsilon_{2}\left(\hat{r}_{i j}, e_{i}, e_{j}\right)\right]^{\mu},
$$

where the exponents $v$ and $\mu$ are the adjustable parameter, and

$$
\varepsilon_{1}\left(e_{i}, e_{j}\right)=\left[1-\chi^{2}\left(e_{i} \cdot e_{j}\right)^{2}\right]^{-1 / 2},
$$

and

$$
\begin{aligned}
& \varepsilon_{2}\left(\hat{r}_{i j}, e_{i}, e_{j}\right)= \\
& 1-\frac{\chi^{\prime}}{2}\left[\frac{\left(e_{i} \cdot \hat{r}_{i j}+e_{j} \cdot \hat{r}_{i j}\right)^{2}}{1+\chi^{\prime}\left(e_{i} \cdot e_{j}\right)}+\frac{\left(e_{i} \cdot \hat{r}_{i j}-e_{j} \cdot \hat{r}_{i j}\right)^{2}}{1-\chi^{\prime}\left(e_{i} \cdot e_{j}\right)}\right]
\end{aligned}
$$

with $\chi^{\prime}=\left(\kappa^{1 / \mu}-1\right) /\left(\kappa^{1 / \mu}+1\right)$. Here $\kappa=\sigma_{e e} / \sigma_{s s}$ is the aspect ratio of the ellipsoid of revolution with $\sigma_{e e}$ denoting the separation between two ellipsoids of revolution in a end-to-end configuration, and $\sigma_{s s}=\sigma_{0}$, and $\kappa^{\prime}=\varepsilon_{s s} / \varepsilon_{e e}$, where $\varepsilon_{s s}$ is the depth of the minimum of the potential for a pair of ellipsoids of revolution aligned in a side-by-side configuration, and $\varepsilon_{e e}$ is the corresponding depth for the end-to-end alignment. Here $\varepsilon_{0}$ is the depth of the minimum of the pair potential between two ellipsoids of revolution aligned in cross configuration. The GB pair potential defines a family of models, each member of which is characterized by the values chosen for the set of four parameters $\kappa, \kappa^{\prime}, \mu$, and $\nu$, and is represented by GB $\left(\kappa, \kappa^{\prime}, \mu, v\right)^{7}$. Systems consist of 500 ellipsoids of revolution in a cubic box with periodic boundary conditions at several temperatures, starting from the high-temperature isotropic phase down to the nematic phase across the I-N phase boundary have been simulated. We have carried out several simulations with different aspect ratios $(\kappa)$ where for each aspect ratio isochors of different densities have been investigated. All quantities are given in reduced units defined in terms of the Gay-Berne potential parameters $\varepsilon_{0}$ and $\sigma_{0}$ : length in units of $\sigma_{0}$, temperature in units of $\varepsilon_{0} / k_{B}$, and time in units of $\left(\sigma_{0}^{2} m / \varepsilon_{0}\right)^{1 / 2}$, $m$ being the mass of the ellipsoids of revolution. The mass as well as the moment of inertia of each of the ellipsoids of revolution have been set equal to unity. The intermolecular potential is truncated at a distance $r_{\text {cut }}$ and shifted such that $U\left(r_{i j}=r_{\text {cut }}\right)=0, r_{i j}$ being the separation between two ellipsoids of revolution $i$ and $j$. The equations of motion have been integrated using the velocity-verlet algorithm with integration time step $d t=0 \cdot 0015 .^{8}$ Equilibration has been done by periodic re-scaling of linear and angular velocities of particles. This has been done for a time period of $t_{q}$ following which the system has been allowed to 
propagate with a constant energy for a time period of $t_{e}$ in order to ensure equilibration upon observation of no drift of temperature, pressure, and potential energy. The data collection has been executed in a microcanonical ensemble. At each state point, local potential energy minimization has been executed by the conjugate gradient technique for a subset of 200 statistically independent configurations. The landscape analysis has been done with a system size of 256 ellipsoids of revolution, which is big enough for having no qualitative change in the results due to the system size. ${ }^{9}$ Minimization has been performed with three position coordinates and two Euler angles for each particle, the third Euler angle being redundant for ellipsoids of revolution.

\section{Results and discussion}

\subsection{Single particle orientational dynamics}

The orientational dynamics of the system at the single particle level may be described by the first and second order single particle orientational time correlation functions (OTCF) $C_{l}^{s}(t)(l=1,2)$, which are defined by

$$
C_{l}^{S}(t)=\frac{\left\langle\sum_{i} P_{l}\left(e_{i}(t) \cdot e_{i}(0)\right)\right\rangle}{\left\langle\sum_{i} P_{i}\left(e_{i}(0) \cdot e_{i}(0)\right)\right\rangle},
$$

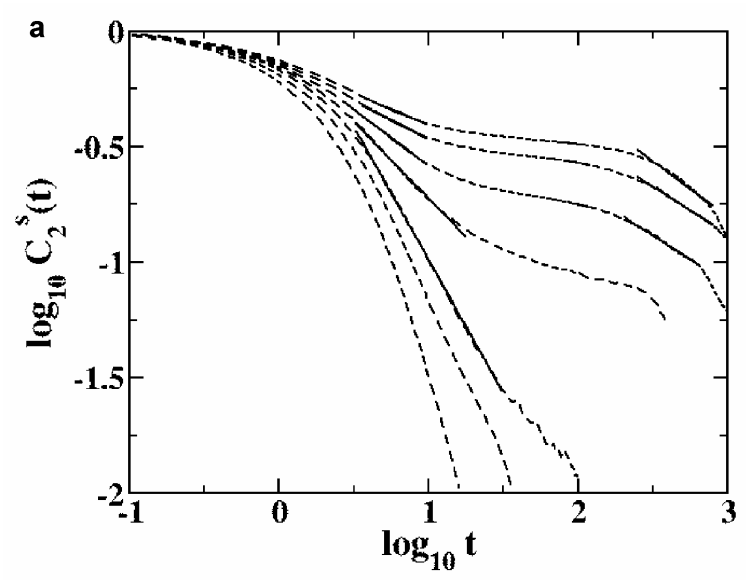

where $P_{l}$ is the $l$-th rank Legendre polynomial and the angular brackets stand for ensemble averaging. Figure 1a shows the single particle second rank OTCF in a log-log plot as the temperature is lowered from high temperature isotropic phase to low temperature nematic phase across the I-N transition. The I-N transition is marked by a jump in the orientational order parameter $\mathrm{S}$, defined for an $\mathrm{N}$ particle system as the largest eigenvalue of the ordering matrix $\mathrm{Q}$ :

$$
Q_{\alpha \beta}=\frac{1}{N} \sum_{i=1}^{N} \frac{1}{2}\left(3 e_{i \alpha} e_{i \beta}-\delta_{\alpha \beta}\right)
$$

where $e_{i \alpha}$ is the $\alpha$-component (in the space-fixed frame) of the unit orientation vector $e_{i}$ along the principal symmetry axis of the $i$ th ellipsoid of revolution. ${ }^{10}$ Note the emergence of the power law decay at short to intermediate times near the $\mathrm{I}-\mathrm{N}$ phase boundary. As the I-N phase boundary is crossed upon cooling, the advent of two power law decay regimes separated by an intervening plateau at shortto-intermediate times imparts a step-like feature to the temporal behaviour of the second rank OTCF. Such power law relaxation near I-N phase boundary was an area of great interest in the recent past ${ }^{11-16}$ and it has been investigated that the scenario is not a unique property of the model we have studied; it is a rather universal phenomenon of second rank OTCF. ${ }^{17}$

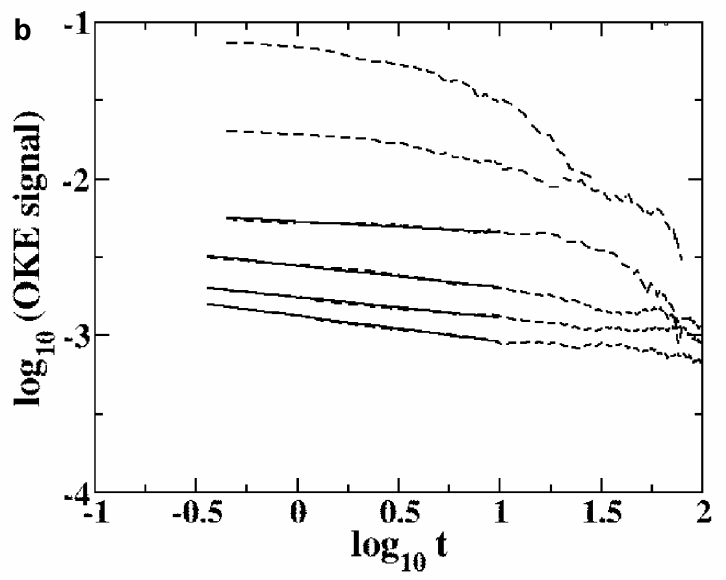

Figure 1. The time evolution of the (a) single particle OTCF on a log-log plot for GB $(3,5,2,1)$ along an isochor with density $\rho=0.32$ across I-N transition at temperatures $T=2.008,1.697,1.499,1.396,1.310$, 1.199 and 1.102 from left to right and (b) collective second rank OTCF for the same system and isochor at temperatures $T=2.008,1.499,1.396,1.310,1.199$ and 1.102 form top to bottom. $T_{\mathrm{I}-\mathrm{N}}$ is located between $T=1.499$ and $T=1 \cdot 396$. The portions fitted with straight line correspond to power law decay regime. 

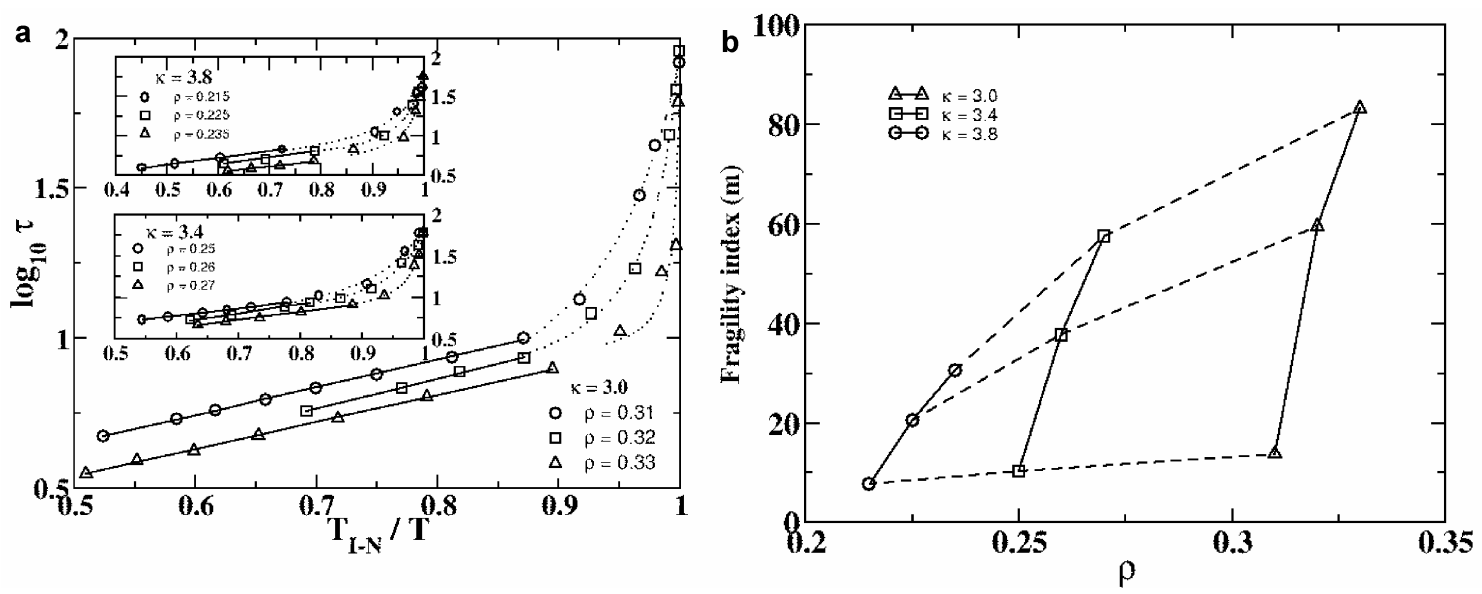

Figure 2. (a) The orientational correlation time in the logarithmic scale as function of the inverse of the scaled temperature, the scaling being done by the isotropic to nematic transition temperature $T_{\mathrm{I}-\mathrm{N}}$. For the insets, the horizontal and the vertical axis labels read same as that of the main frame and are thus omitted for clarity. Along each isochor, the solid line is the Arrhenius fit to the subset of the high-temperature data and the dotted line corresponds to the fit to the data near the isotropic-nematic phase boundary with the VFT form. (b) The fragility index m shown as a function of density for different aspect ratios. The dashed lines are guide to the eye to illustrate the fact that the dependence of the fragility index on the density is becoming stronger as the aspect ratio becomes smaller.

Such a feature bears remarkable similarity to what is observed for supercooled liquids as the glass transition is approached from the above. ${ }^{18,19}$ While for the supercooled liquid the emergence of step-like feature is well understood as a consequence of $\beta$ relaxation, the origin of such a feature observed for liquid crystal defied of reliable explanation.

\subsection{Collective orientational dynamics}

In experiments, one can probe orientational relaxation through the decay of the OKE signal, which is given by the negative of the time derivative of the collective second rank OTCF $C_{2}^{c}(t) .^{4,20,21}$ The latter, which is defined by

$$
C_{2}^{c}(t)=\frac{\left\langle\sum_{i} \sum_{j} P_{2}\left(e_{i}(0) \cdot e_{j}(t)\right)\right\rangle}{\left\langle\sum_{i} \sum_{j} P_{2}\left(e_{i}(0) \cdot e_{j}(0)\right)\right\rangle} .
$$

Calculation of this correlation function is computationally demanding, particularly at longer times. In order to set a direct link with experimental results, we show the temporal behaviour of the OKE signal in the $\log -\log$ plot for the system across the $\mathrm{I}-\mathrm{N}$ phase transition in figure $1 \mathrm{~b}$. The short-to-intermediate-time power law regime is evident in the OKE signal for the system studied here. Like single particle second rank OTCF, it is also verified to be a universal phenomenon near I-N transition. ${ }^{17}$

\section{Fragility of liquid crystals}

We estimate the orientational correlation time $\tau$ as the time taken for $C_{2}^{S}(t)$ to decay by $90 \%$, i.e. $C_{2}^{S}(t=\tau)=$ $0 \cdot 1$. Figure $2 \mathrm{a}$ shows $\tau$ in the logarithmic scale as a function of the inverse temperature along the three isochors for each of the three systems considered. We have scaled the temperature by $T_{\mathrm{I}-\mathrm{N}}$ in the spirit of Angell's plot, that displays the shear viscosity (or the structural relaxation time, the inverse diffusivity, etc.) of glass-forming liquids as a function of the inverse of the scaled temperature, the scaling being done in the latter case by the glass transition temperature $T_{g}{ }^{22,23}$ For all the three systems, two distinct features are common: (i) in the isotropic phase far away from the $\mathrm{I}-\mathrm{N}$ transition, the orientational correlation time $\tau$ exhibits the Arrhenius temperature dependence, i.e. $\tau(T)=\tau_{0} \exp \left(E / k_{B} T\right)$, where the activation energy $E$ and the pre-factor $\tau_{0}$ are both independent of temperature; (ii) in the isotropic phase near the I-N transition, the temperature dependence of $\tau$ shows marked deviation from the Arrhenius behaviour and can be well described by the VogelFulcher-Tammann (VFT) equation $\tau(T)=\tau_{0} \exp [B /$ 
$\left.\left(T-T_{\mathrm{VFT}}\right)\right]$, where $\tau_{0}, B$, and $T_{\mathrm{VFT}}$ are constants, independent of temperature. Again these features bear remarkable similarity with those observed for fragile glass-forming liquid. A non-Arrhenius temperature behaviour is taken to be the signature of fragile liquids. For fragile liquids, the temperature dependence of the shear viscosity follows the Arrhenius behaviour far above $T_{g}$ and can be fitted to the VFT functional form in the deeply supercooled regime near $T_{g}{ }^{22,23}$ The striking resemblance in the dynamical behaviour described above between the isotropic phase of thermotropic liquid crystals near the I-N transition and supercooled liquids near the glass transition has prompted us to attempt a quantitative measure of glassy behaviour near the I-N transition. For supercooled liquids, one quantifies the dynamics by a parameter called fragility index which measures the rapidity at which the liquid's properties (such as viscosity) change as the glassy state is approached. In the same spirit ${ }^{24}$ that offers a quantitative estimation of the fragile behaviour of supercooled liquids, we here define the fragility index $m$ of a thermotropic liquid crystalline system as ${ }^{16}$

$$
m=\left.\frac{d \log _{10} \tau(T)}{d\left(T_{\mathrm{I}-\mathrm{N}} / T\right)}\right|_{T=T_{\mathrm{I}-\mathrm{N}}} .
$$

It is clear from the above equation that if $\tau(T)$ follows Arrhenius temperature dependence, $\mathrm{m}$ will be constant throughout the whole temperature range. Figure $2 \mathrm{~b}$ shows the density dependence of the fragility index for the three systems with different aspect ratios. For a given aspect ratio, the fragility index increases with increasing density, the numerical values of the fragility index $m$ being comparable to those of supercooled liquids. The density dependence observed in the present work is remarkably similar to those observed for supercooled liquids. For the range of aspect ratios studied here, the dependence of the fragility index on the density is becoming stronger as the aspect ratio becomes smaller.

\section{Heterogeneous dynamics}

Another hallmark of fragile glass-forming liquids is spatially heterogeneous dynamics ${ }^{25}$ reflected in nonGaussian dynamical behaviour. ${ }^{26}$ It is intuitive that the growth of the pseudo-nematic domains, characterized by local nematic order, in the isotropic phase near the I-N transition would result in heterogeneous dynamics in liquid crystals. We have, therefore, monitored the time evolution of the rotational nonGaussian parameter (NGP), ${ }^{27,28} \alpha_{2}^{R}(t)$, which in the present case is defined as

$$
\alpha_{2}^{R}(t)=\frac{\left\langle\Delta \phi^{4}(t)\right\rangle}{2\left\langle\Delta \phi^{2}(t)\right\rangle^{2}}-1,
$$

where

$$
\left\langle\Delta \phi^{2 n}(t)\right\rangle=\frac{1}{N} \sum_{i=1}^{N}\left\langle\left|\phi_{i}(t)-\phi_{i}(0)\right|^{2 n}\right\rangle .
$$

Here $\phi_{i i}$ is the rotation vector like the position vector $r_{i}$ appears in the case of translational NGP of $i$ th ellipsoid of revolution, the change of which is defined by

$$
\Delta \phi_{i}(t)=\phi_{i}(t)-\phi_{i}(0)=\int_{0}^{t} d t^{\prime} \omega\left(t^{\prime}\right),
$$

$\omega_{i}$ being the corresponding angular velocity, ${ }^{18,19}$ and $N$ is the number of ellipsoids of revolution in the system. NGP will have value equal to zero when system dynamics is spatially homogeneous and will have a non-zero value when the system dynamics is spatially heterogeneous. As a typical behaviour, figure $3 \mathrm{a}$ and $\mathrm{b}$ show the time dependence of the rotational NGP for one of the systems at several temperatures across the I-N transition along an isochor. On approaching the I-N transition upon cooling, a bimodal feature starts appearing with the growth of a second peak, which eventually becomes the dominant one, at longer times. ${ }^{16,27} \mathrm{We}$ further investigate the appearance of this bimodal feature in NGP plot. To this end we calculate mean square angular deviation (MSAD) of the system at different temperatures starting from high temperature isotropic phase to low temperature nematic phase. The appearance of the bimodal feature in the rotational NGP is accompanied by a signature of a sub-diffusive regime in the temporal evolution of the MSAD, the time scale of the short-time peak and that of the onset of the subdiffusive regime being comparable, as shown in figure $3 \mathrm{a}^{16,27}$ We note that the dominant peak appears on a time scale which is comparable to that of onset of the diffusive motion in orientational degrees of freedom (ODOF) as evident in figure 3a. ${ }^{16,27}$ Similar feature has been observed recently for supercooled water. ${ }^{28} \mathrm{We}$ further find that the time scale at which 


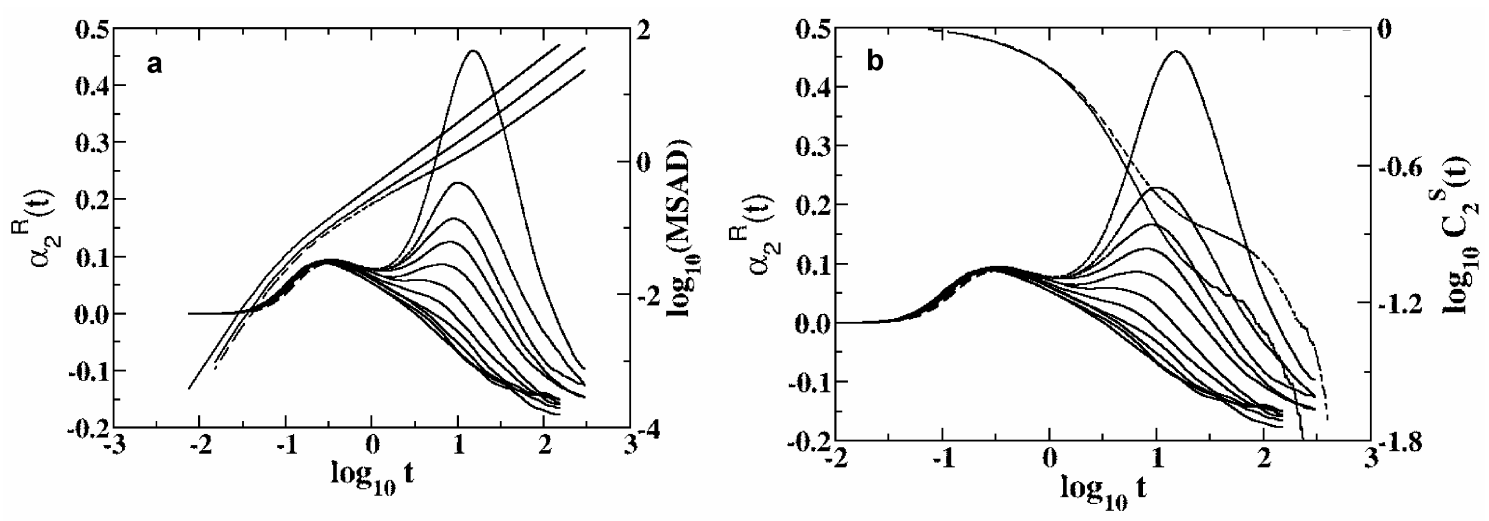

Figure 3. Time evolution of the rotational non-Gaussian parameter $\alpha_{2}^{R}(t)$ in a semi-log plot for the system with aspect ratio $\kappa=3$. The time dependence is shown at several temperatures $(T=3 \cdot 5,3 \cdot 25,3 \cdot 0,2 \cdot 75,2 \cdot 5$, $2 \cdot 25,2.0,1.88,1.82,1.78$ and 1.5) across the isotropic-nematic (I-N) transition along an isochor at density $=$ 0.33. (a) On a different scale along the vertical axis (appearing on the right), time evolution of the mean square angular deviation $\left\langle\Delta \phi^{2}(t)\right\rangle$ is shown in a log-log plot for three temperatures: the highest temperature studied in the isotropic phase $(T=1.5)$ and the other two temperatures $(T=3.0$ and 2.75$)$ that are closest to the I-N transition on either side along with the time evolution of $\alpha_{2}^{R}(t)$, and (b) On a different scale along the vertical axis (appearing on the right), the time evolution of the single-particle second rank orientational time correlation function $C_{2}^{S}(t)$ is shown in a log-log plot for the two temperatures $(T=3.0$ and 2.75$)$ that are closest to the I-N transition on either side along with the time evolution of $\alpha_{2}^{R}(t)$. The solid lines denote the curves for the high temperature isotropic phase and the dashed lines for the low temperature nematic phase.

the long-time peak appears is also comparable to the time scale of onset of the plateau that is observed in the time evolution of $C_{2}^{S}(t)$, as shown in figure $3 b .^{16,27}$

\section{Energy landscape analysis}

In recent years, energy landscape analysis has gained considerable importance as a powerful approach to understand pathways of protein folding. At the same time, several studies have achieved significant success in interpreting the anomalous dynamics observed in many glass-forming liquids in terms of the features of the underlying energy landscapes. ${ }^{29-34}$ Energy landscape analysis gives the potential energy, which devoid of any kind of thermal motions, of inherent structures of the parent liquid and hence provides a better understanding of the structure and dynamics of the parent liquid. Figure 4 a displays the average inherent structure energy as the change in temperature drives the system across the mesophases along three different isochors. Figure $4 \mathrm{~b}$ shows the concomitant evolution of the average orientational order parameter $S$ both for the inherent structures and the corresponding pre-quenched ones. It is evident that the average inherent structure energy remains fairly insensitive to temperature in the isotropic phase before it starts undergoing a steady fall below a certain temperature that corresponds to the onset of the growth of the orientational order. ${ }^{9}$ As the orientational order grows through the nematic phase, the system continues to explore deeper potential energy minima until a plateau is reached on arrival at the smectic phase. ${ }^{9}$ In the inset of figure $4 a$, the location of the maximum of the mean square fluctuation in the inherent structure energy shows that the system explores potential energy minima spanning over a broader energy range as it settles into the nematic phase. This suggests the critical role of fluctuation effects in the nematic phase. The average potential energy for a state point obtained from the molecular dynamics trajectory, however, decreases rather smoothly in all three phases with decrease in temperature as illustrated in the inset of figure $4 \mathrm{~b}$. It is evident that the signature of the $\mathrm{I}-\mathrm{N}$ transition is quite weak here in contrast to that of the nematicsmectic transition. We have repeated the same analysis for a larger system size to check the effect of finite system size, but qualitatively ended up with same conclusions as the smaller one. Note that this has been observed for a glassy system, ${ }^{32}$ where the average IS energy also falls over a temperature range. ${ }^{30}$ 

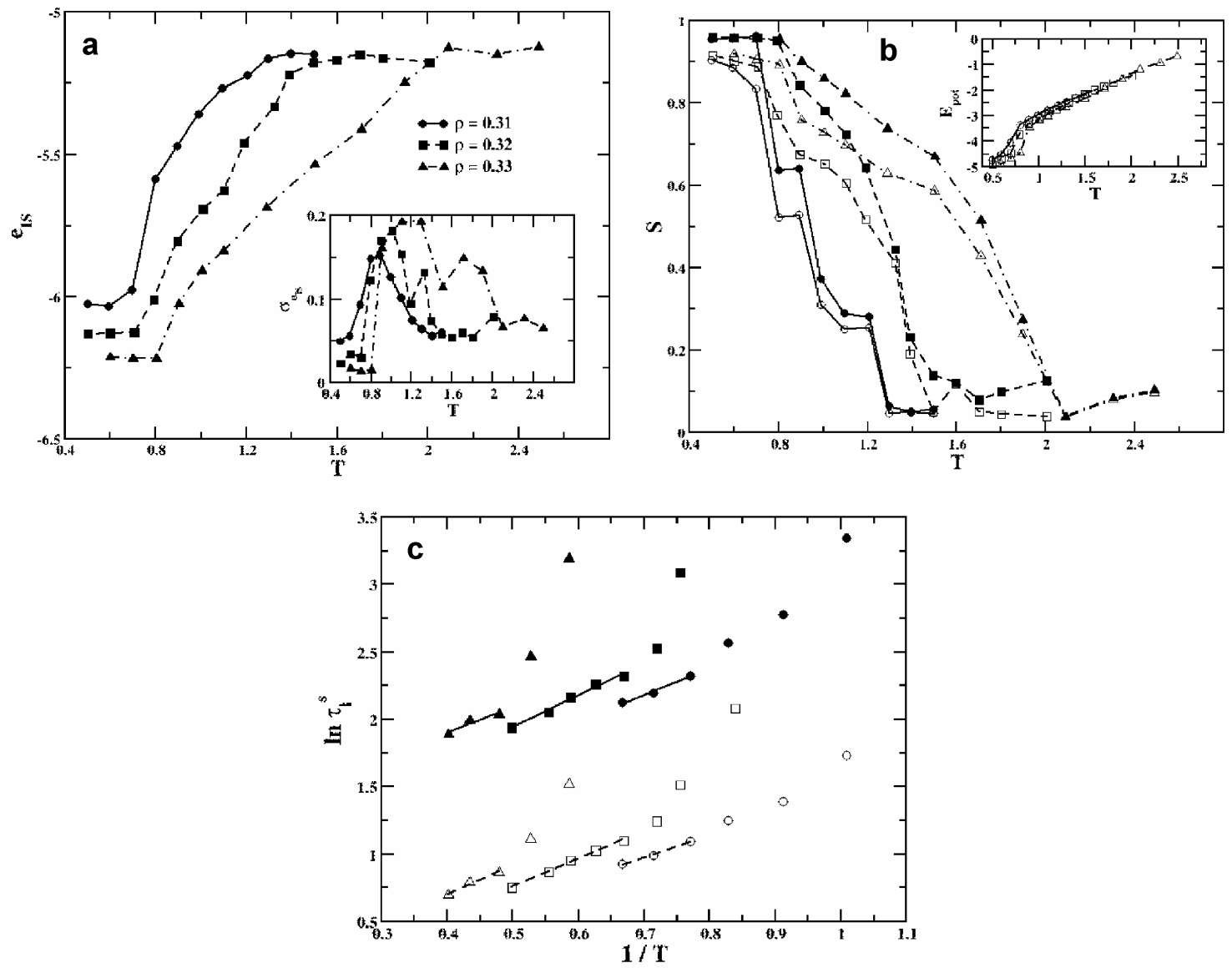

Figure 4. (a) The temperature dependence of the average inherent structure energy per particle $e_{I S}$ along three isochors at densities $\rho=0 \cdot 31,0.32$, and 0.33 for $\kappa=3$. The inset shows the root mean square fluctuation in inherent structure energy $\sigma_{\mathrm{e}}$, computed from a subset of 200 configurations for each state point, as a function of temperature $T$ at the same three densities. (b) The evolution of the average order parameter $\mathrm{S}$ with temperature both for the inherent structures (filled) and the corresponding pre-quenched ones (empty). The inset shows the temperature dependence of the average potential energy $E_{\text {pot }}$ at a state point obtained from averaging over the molecular dynamics trajectory. For clarity, $E_{\mathrm{pot}}$ is shown for the state points along only one isochor corresponding to the density $\rho=0 \cdot 32$. The state points considered in our simulations correspond to (i) the isotropic $(I)$ phase for $T \geq 1.297$ and the smectic-B (Sm-B) phase for $T \leq 0.595$ along the isochor at $\rho=0.31$; (ii) $I$ for $T \geq 1.495$ and Sm-B for $T \leq 0.706$ at $\rho=0.32$; (iii) $I$ for $T \geq 2.089$ and Sm-B for $T \leq 0.803$ at $\rho=0.33$. (c) The inverse temperature dependence of the singleparticle orientational relaxation times $\tau_{l}^{S}, l=1$ (filled) and $l=2$ (empty), in the logarithmic scale. The straight lines are the Arrhenius fits for the subsets of data points, each set corresponding to a fixed density: $\rho=0.31$ (circle), $\rho=0.32$ (square), $\rho=0.33$ (triangle up).

Like supercooled liquid, we have also observed a Gaussian form of number density of IS with $e_{\mathrm{IS}}{ }^{16}$

Figure $4 \mathrm{c}$ illustrates the correlation of the energy landscape behaviour with the dynamics the system exhibits. Here, we define relaxation times $\tau_{l}^{S}(T)$ as the time when $C_{l}^{S}(t)=e^{-1}{ }^{9}$. The dramatic slow down of orientational dynamics with decreasing temperature near the I-N transition manifests in the temperature dependence of these relaxation times. Figure $4 \mathrm{c}$ shows that in the isotropic phase far from the $\mathrm{I}-\mathrm{N}$ transition region $\tau_{l}^{S}(T)$ exhibits the Arrhenius behaviour, i.e. $\tau_{l}^{S}(T)=\tau_{0, l} \exp \left[E_{l} /\left(k_{B} T\right)\right]$, where the activation energy $E_{l}$ and the infinite temperature relaxation time $\tau_{0, l}$ are independent of temperature. We find that the breakdown of the Arrhenius behaviour occurs at a temperature that marks the onset of the growth of the depth of the potential energy minima explored by the system. ${ }^{9}$ Such correlations of different other pro- 
perties with the landscape have been investigated in several other studies for both supercooled liquids and thermotropic liquid crystals. ${ }^{35,36}$

\section{Conclusion}

Computer simulation studies of single particle and collective orientational dynamics of thermotropic liquid crystals near the isotropic-nematic $(\mathrm{I}-\mathrm{N})$ transition are presented and compared with the dynamics of supercooled liquids near glass transition. The short-to-intermediate time scale power law decay in the orientational relaxation appeared to be the most intriguing feature. In analogy with the supercooled liquids, a fragility index of liquid crystals is introduced to quantify the glassiness of orientational dynamics near the $\mathrm{I}-\mathrm{N}$ transition. Our investigation of spatially heterogeneous dynamics strengthens the analogy further. The striking resemblance in the correspondence between the manner of the exploration of the potential energy landscape and the onset of the non-Arrhenius temperature dependence of the relaxation time might imply a unique underlying landscape mechanism for slow dynamics in soft condensed matter. The close analogies in the dynamics as well as in the features of energy landscape explored here may suggest that there could be an avoided second order thermodynamic phase transition underlying both the $\mathrm{I}-\mathrm{N}$ and the glass transition.

\section{Acknowledgements}

We thank Dr Dwaipayan Chakrabarti for helpful suggestions and discussions during the preparation of the manuscript. We also thank Dr Prasanth P Jose for collaboration during the early part of the study. This work was supported in parts by grants from Department of Science and Technology (DST) and Council of Scientific and Industrial Research (CSIR). B J thanks CSIR for providing JRF.

\section{References}

1. de Gennes P G and Prost J 1993 The physics of liquid crystals (Oxford: Clarendon Press)

2. Chandrasekhar S 1992 Liquid crystals (Cambridge: Cambridge University Press)

3. Gottke S D, Bruce D D, Cang H, Bagchi B and Fayer M D 2002 J. Chem. Phys. 116360

4. Gottke S D, Cang H, Bagchi B and Fayer M D 2002 J. Chem. Phys. 1166339
5. Cang H, Li J, Novikov V N and Fayer M D 2003 J. Chem. Phys. 1189303

6. Gay J G and Berne B J 1981 J. Chem. Phys. 743316

7. Bates M A and Luckhurst G R 1999 J. Chem. Phys. 1107087

8. Ilnytskyi J M and Wilson M R 2002 Comput. Phys. Commun. 14843

9. Chakrabarti D and Bagchi B 2006 Proc. Natl. Acad. Sci. USA 1037217

10. Zannoni C 2000 in Advances in the computer simulations of liquid crystals (eds) P Pasini and C Zannoni (Dordrecht: Kluwer Academic Publishers) pp. 17-50

11. Jose P P and Bagchi B 2004 J. Chem. Phys. 12011256

12. Jose P P and Bagchi B 2006 J. Chem. Phys. 125 184901

13. Chakrabarty S, Chakrabarti D and Bagchi B 2006 Phys. Rev. E73 061706

14. Chakrabarti D, Jana B and Bagchi B 2007 Phys. Rev. E75 061703

15. Chakrabarti D and Bagchi B 2007 J. Chem. Phys. 126 204906

16. Jana B, Chakrabarti D and Bagchi B 2007 Phys. Rev. E76 0711712

17. Chakrabarti D, Jose P P, Chakrabarty S and Bagchi B 2005 Phys. Rev. Lett. 95197801

18. Kämmerer S, Kob W and Schilling R 1997 Phys. Rev. E56 5450

19. Michele C De and Leporini D 2001 Phys. Rev. E63 036702

20. (a) Yan Y-X and Nelson K A 1987 J. Chem. Phys. 87 6240; (b) Yan Y-X and Nelson K A 1987 J. Chem. Phys. 876257

21. Li J, Wang I and Fayer M D 2005 J. Phys. Chem. B109 6514

22. Angell C A 1988 J. Phys. Chem. Solids. 49863

23. Angell C A 1991 J. Non-Cryst. Solids 131-133 13

24. Böhmer R, Ngai K L, Angell, C A and Plazek D J 1993 J. Chem. Phys. 994201

25. Ediger M D 2000 Ann. Rev. Phys. Chem. 5199

26. Shell M S, Debenedetti P G and Stillinger F H 2005 J. Phys.: Condens. Matter 17 S4035

27. Jose P P, Chakrabarti D and Bagchi B 2006 Phys. Rev. E71 030701(R)

28. Mazza M G, Giovambattista N, Starr F W and Stanley H E 2006 Phys. Rev. Lett. 96057803

29. Wales D J 2003 Energy landscapes (Cambridge: Cambridge University Press)

30. Sastry S, Debenedetti P G and Stillinger F H 1998 Nature (London) 393554

31. Scala A, Starr F W, Nave E L, Sciortino F and Stanley H E 2000 Nature (London) 406166

32. Sastry S 2001 Nature (London) 409164

33. Martinez L-M and Angell C A 2001 Nature (London) 410633

34. Salka-Voivod I, Poole P H and Sciortino F $2001 \mathrm{Na}$ ture (London) 412514

35. Chakrabarti D and Bagchi B 2006 Phys. Rev. Lett. 96 187801

36. Chakrabarti D and Bagchi B 2006 Phys. Rev. E74 041704 